\title{
Nonlinear Blind Equalizers: NCMA and NMCMA
}

\author{
Donglin Wang, Sandeep Chandana \\ Department of Electrical and Computer Engineering, University of Calgary, Calgary, Canada \\ E-mail: \{dowang, schandan\} @ucalgary.ca \\ Received March 5, 2010; revised April 19, 2010; accepted May 20, 2010
}

\begin{abstract}
This paper proposes two nonlinear blind equalizers: the nonlinear constant modulus algorithm (NCMA) and the nonlinear modified constant modulus algorithm (NCMA) by applying a nonlinear transfer function (NTF) into constant modulus algorithm (CMA) and modified constant modulus algorithm (MCMA), respectively. The effect of the NTF on CMA and MCMA is theoretically analyzed, which implies that the NTF can make their decision regions much sharper so that the proposed two nonlinear blind equalizers are more robust against the convergency error compared to their linear counterparts. The embedded single layer in NCMA and NMCMA simultaneously guarantees a comparably speedy convergency. On 16-quadrature amplitude modulation (QAM) symbols, computer simulations show that NCMA achieves an 8dB lower convergency mean square error (MSE) than CMA, and NMCMA achieves a 15dB lower convergency MSE than MCMA.
\end{abstract}

Keywords: Nonlinear Blind Equalizer, Nonlinear Transfer Function (NTF), CMA, Nonlinear CMA (NCMA), Nonlinear MCMA (NMCMA)

\section{Introduction}

Constant modulus algorithm (CMA) [1-6] is widely used for blind equalization [2,5,6,7-10] for constant modulus transmissions in communication systems in order to overcome the propagation channel corruption, mitigate the inter-symbol interference (ISI) and recover the transmitted symbols, which usually has a satisfied performance in common situations. However, under a complicated multipath channel, the transmitted symbols suffer from severe distortion and CMA will perform poor for multi-modulus symbols, i.e. for high-order quadrature amplitude modulation (QAM) symbols, mainly due to the inability of CMA on phase error correction [11].

To suppress the convergence error and improve the equalization performance for multi-modulus symbols, [11-14] proposed a classical modified constant modulus algorithm (MCMA), in which the real component and the imaginary component of the equalizer output are respectively considered to compress the phase error, leading to a better performance. However, its performance is not good enough in some severe cases, since its decision region is comparably smooth, which does not tolerate the convergency error very much.

The method for further improvement is to bring in nonlinearity instead of linearity, which can be realized by utilizing multilayer architecture, nonlinear transfer function
(NTF) [15] or neural network [16-18]. However, as a tradeoff, the complicated multiple architecture results in a slower convergency. As we all know, the speedy convergency is significant for adaptive blind equalization. Consequently, in this paper, we preferentially consider introducing a NTF into blind equalization to improve the performance. A NTF, $f(x)=x+\alpha \sin (\pi x)$, is proposed in [18] for blind equalizer according to its provided properties. However, there is a remaining unsolved question: in essence, why can this NTF be helpful for equalization performance? Or equivalently, what is the theoretical effect of NTF on equalization performance? This paper will answer this question via theoretical analysis. The following theoretical derivation provides that the NTF can make the decision region much sharper so that the proposed nonlinear blind equalizers are more robust against the convergency error. Based on this discovery, by applying the nonlinear transfer function (NTF) to CMA and MCMA, the nonlinear CMA (NCMA) and nonlinear modified CMA (NMCMA) are thus proposed, and their adaptive learning rules are also theoretically derived in this paper.

The remainder of this paper is organized as follows. Section II theoretically analyzes the effect of the NTF on blind equalizers. Based on the analysis given in Section 2, the nonlinear blind equalizer, NCMA, is proposed in Section III. Moreover, another blind equalizer, NMCMA, is proposed in Section 4. Simulation results of the proposed 
nonlinear blind equalizers on 16-QAM symbols are reported in Section 5, which are compared to their linear counterparts, followed by conclusion in Section 6 .

Notation: $E[\cdot]$ represents expectation of a random variable. $|\cdot|$ denotes the modulus of a complex number or the absolute value of a real number. The superscripts $(\cdot)^{*}$ and $(\cdot)^{\prime}$ denote the conjugate and derivative, respectively. Both $\operatorname{Re}\{\cdot\}$ and $(\cdot)_{R}$ denote the real component while both $\operatorname{Im}\{\cdot\}$ and $(\cdot)_{I}$ denote the imaginary component.

\section{Effect of NTF}

\subsection{CMA}

The cost function of CMA can be expressed as [1]

$$
J_{C M A}(n)=\frac{1}{4} E\left[\left(|y(n)|^{2}-R_{2}\right)^{2}\right]
$$

where $n$ denotes the time index; $y(n)$ represents the equalized symbol; assuming that $s(n)$ is the complex-valued transmitted symbol, the constant modulus, $R_{2}$, is given by $R_{2}=\frac{E\left[|S(n)|^{4}\right]}{E\left[|S(n)|^{2}\right]}$. The 3D performance function of CMA, $J_{C M A}$, is shown versus $y(n)$ in [1].

\subsection{Performance Function without NTF}

Considering the case without a NTF in CMA [1],

$$
y(n)=X^{T}(n) W=\sum_{m=0}^{M-1} x(n-m) w_{m}
$$

where, $X(n)=[x(n), x(n-1), \cdots, x(n-M+1)]^{T}$ is the corrupted signal at the receiver, which is also the input signal of the equalizer, and $W=\left[w_{0}, w_{1}, \cdots, w_{M-1}\right]^{T}$ is the adaptive weight vector. Assuming keeping all weights unchanged except $w_{p}, 0 \leq p \leq M-1$, (2) can be expressed as

$$
\begin{aligned}
y(n) & =\sum_{m=0, m \neq p}^{M-1} x(n-m) w_{m}+x(n-p) w_{p} \\
& =C+x(n-p) w_{p}
\end{aligned}
$$

where $C=\sum_{m=0, m \neq p}^{M-1} x(n-m) w_{m}$ is a constant in this case. Using (3) together with (1), the 2D performance function, $J_{C M A}$, is shown versus $\operatorname{Re}\left\{w_{p}\right\}$ in Figure 1, similar as

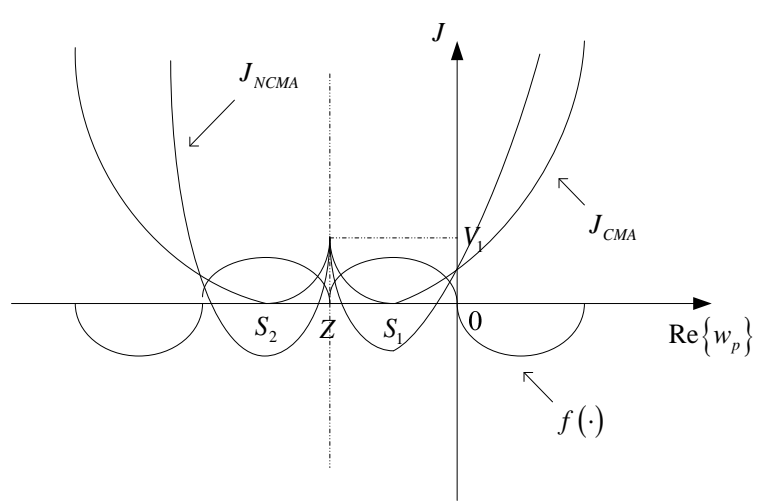

Figure 1. Comparison of performance functions, $J$, versus $\operatorname{Re}\left\{w_{p}\right\}$, where $f(\bullet)$ represents the NTF, $S_{1}$ and $S_{2}$ correspond to two stable minimum values and $V_{1}$ corresponds to an unstable local maximum.

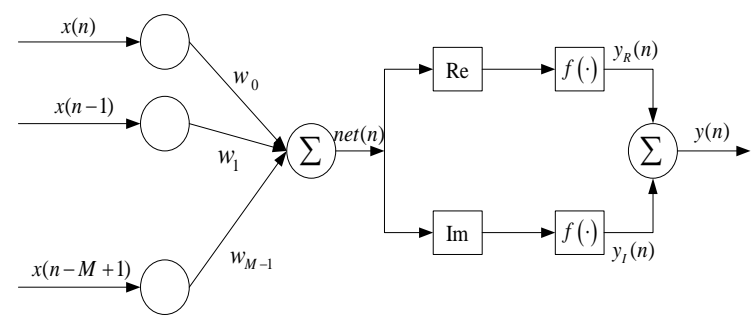

Figure 2. Architecture representation for the proposed nonlinear blind equalizers: NCMA and NMCMA, where it is noticed that the real component and the imaginary component of the received symbols are separately considered.

$J_{C M A}$ versus $y(n)$ in [1]. In Figure 2, two stable points, $S_{1}$ and $S_{2}$, are given by $S_{1}=$ $\operatorname{Re}\left\{\frac{-\sqrt{R_{2}} e^{j \theta}-C}{x(n-p)}\right\}$ and $S_{2}=\operatorname{Re}\left\{\frac{\sqrt{R_{2}} e^{j \theta}-C}{x(n-p)}\right\}$, respectively, $\theta$ denotes an arbitrary phase; $Z=\operatorname{Re}$ $\left\{\frac{-C}{x(n-p)}\right\}$; and the value at $Z$ is given by $V_{1}=\frac{R_{2}^{2}}{4}$.

\subsection{Performance Function with NTF}

Let us consider the blind equalizer with a NTF, i.e. NCMA. Define its corresponding cost function by $J_{\text {NCMA }}(n) \triangleq J_{\text {CMA }}(n)$, i.e.

$$
J_{\text {NCMA }}(n)=J_{\text {CMA }}(n)=\frac{1}{4} E\left[\left(|y(n)|^{2}-R_{2}\right)^{2}\right]
$$

Without loss of generality, a NTF with the expression of $f(x)=x+\alpha \sin (2 \pi f x)$, where $\alpha$ is a nonlinear 
coefficient and $f$ denotes the frequency of the sine function, is considered in this paper for performance analysis. With this NTF, based on the proposed architecture as shown in Figure 2, we have

$$
\begin{aligned}
y(n) & =f\left(\operatorname{Re}\left\{X^{T}(n) W\right\}\right)+j f\left(\operatorname{Im}\left\{X^{T}(n) W\right\}\right) \\
& =C+x(n-p) w_{p} \\
& +\alpha \sin \left(2 \pi f \operatorname{Re}\left\{C+x(n-p) w_{p}\right\}\right) \\
& +j \alpha \sin \left(2 \pi f \operatorname{Im}\left\{C+x(n-p) w_{p}\right\}\right)
\end{aligned}
$$

Since the performance function is symmetrical around the $J$-axis, i.e. the cost function is independent with the phase of $y(n)$ or $w_{p}$, without loss of generality, let us consider the simplest case, i.e. $w_{p}$ is real. In this case, (4) can be approximated as

$$
\begin{aligned}
\frac{y(n)-C}{x(n-p)} & =w_{p}+\frac{\alpha-C}{x(n-p)} \sin \left(2 \pi f \operatorname{Re}\left\{C+x(n-p) w_{p}\right\}\right) \\
& +j \frac{\alpha-C}{x(n-p)} \sin \left(2 \pi f \operatorname{Im}\left\{C+x(n-p) w_{p}\right\}\right) \\
& \stackrel{(a)}{\approx} w_{p}+\frac{\alpha-C}{x(n-p)} \sin \left(2 \pi f \operatorname{Re}\left\{C+x(n-p) w_{p}\right\}\right) \\
& \stackrel{(b)}{\approx} w_{p}+\frac{\alpha-C}{x(n-p)} \sin \left(2 \pi f|x(n-p)| w_{p}\right)
\end{aligned}
$$

where, the approximation ( $a$ ) is based on the fact that the closer the directions of two vectors with fixed modules, the bigger their summation, and the approximation (b) is true because $C$ in (5) is a constant, same as that in (3) and the value of the sine function in (5) is determined by the item containing $w_{p}$. Furthermore, we have

$$
y(n) \approx\left[C+x(n-p) w_{p}\right]+\alpha \sin \left(2 \pi f|x(n-p)| w_{p}\right)
$$

Based on (6) together with (1), the 2D performance function, $J_{N C M A}$, is shown versus $\operatorname{Re}\left\{w_{p}\right\}$ in Figure 1, compared to that without NTF. One can see that using this NTF, its performance function becomes sharper than its previous linear counterpart. Once the convergency point is not exactly the minimum value but one of its surrounding points, namely, there is an estimation bias, i.e. $\delta \mathrm{W}$, its estimation error gets smaller after implanting this NTF. In other words, NCMA is more robust against the convergency error than CMA. It is noticed that, the NTF will provide a good equalization performance under the constraint that $2 \pi f|x(n-p)| \leq \frac{\pi}{2}$, i.e. $0<f \leq \frac{1}{4 \sqrt{R_{2}}}$. Particularly, when $f=\frac{1}{4 \sqrt{R_{2}}}$, the NTF exhibits the optimal equalization performance.

\subsection{Discussion and Extension}

As shown in Figure 1, where the cost function, $J$, is plotted versus the adaptive weights, $\operatorname{Re}\left\{w_{p}\right\}, J_{\text {NCMA }}$ looks similar with $J_{\text {CMA }}$ except that it has a sharper decision region than $J_{C M A}$. The resulting sharper decision region will lead to a better equalization performance in the end. On the other hand, denote by $J_{M C M A}$ and $J_{\text {NMCMA }}$ the cost functions for MCMA and NMCMA, respectively. Since MCMA is similar to CMA except that in NMCA, the real part and the imaginary part are separately considered, after adding a NTF into the linear equalizer, the relationship between $J_{\text {NMCMA }}$ and $J_{M C M A}$ is the same with that between $J_{M C M A}$ and $J_{C M A}$. Therefore, one can know that $J_{\text {NMCMA }}$ also looks similar with $J_{M C M A}$ except that it has a sharper decision region than $J_{M C M A}$.

\section{Proposed NCMA}

In Figure 2, $x(n), x(n-1), \cdots, x(n-M+1)$, as mentioned, are the corrupted symbols at the receiver, $w_{0}, w_{1}, \cdots, w_{M-1}$, as mentioned, are the equalizer taps with the length of $M$, and the variable net is an intermediate variable for convenience. For a time index of $n$, the input and its corresponding output can be formulated as

$$
\operatorname{net}(n)=X^{T}(n) W
$$

and

$$
y(n)=y_{R}(n)+j y_{I}(n),
$$

where

$$
y_{R}(n)=f\left(\operatorname{net}_{R}(n)\right)
$$

and

$$
y_{I}(n)=f\left(\text { net }_{I}(n)\right) .
$$

Furthermore, the real component and the imaginary component of net $(n)$ can be obtained as

$$
n e t_{R}(n)=\sum_{m=0}^{M-1}\left[x_{R}(n-m) w_{m, R}-x_{I}(n-m) w_{m, I}\right]
$$

and

$$
n e t_{I}(n)=\sum_{m=0}^{M-1}\left[x_{R}(n-m) w_{m, I}-x_{I}(n-m) w_{m, R}\right] .
$$

Based on (1), using the statistic gradient descent (SGD) in terms of $w_{p}$, we have 


$$
\begin{aligned}
\frac{\partial J_{N C M A}(n)}{\partial w_{p}} & =\left(|y(n)|^{2}-R_{2}\right)|y(n)| \\
& \times\left(\frac{\partial|y(n)|}{\partial w_{p, R}}+j \frac{\partial|y(n)|}{\partial w_{p, I}}\right),
\end{aligned}
$$

where

$$
\begin{aligned}
\frac{\partial|y(n)|}{\partial w_{p, R}} & =f\left(\operatorname{net}_{R}(n)\right) f^{\prime}\left(\operatorname{net}_{R}(n)\right) x_{R}(n-p) \\
& +f\left(\operatorname{net}_{I}(n)\right) f^{\prime}\left(\operatorname{net}_{I}(n)\right) x_{I}(n-p)
\end{aligned}
$$

and

$$
\begin{aligned}
\frac{\partial|y(n)|}{\partial w_{p, I}} & =f\left(\operatorname{net}_{R}(n)\right) f^{\prime}\left(\operatorname{net}_{R}(n)\right) x_{I}(n-p) \\
& +f\left(\operatorname{net}_{I}(n)\right) f^{\prime}\left(\operatorname{net}_{I}(n)\right) x_{R}(n-p) .
\end{aligned}
$$

Assuming $\mu$ is the learning rate, the weights update from the $n$th step to the $(n+1)$ th step can be expressed as

$$
\begin{aligned}
& W(n+1)=W(n)-\mu e(n) X^{*}(n) \\
& e(n)=\left(|y(n)|^{2}-R_{2}\right)|y(n)| f\left(\operatorname{net}_{R}(n)\right) f^{\prime}\left(\operatorname{net}_{R}(n)\right) \\
& \quad+j\left(|y(n)|^{2}-R_{2}\right)|y(n)| f\left(\operatorname{net}_{I}(n)\right) f^{\prime}\left(\operatorname{net}_{I}(n)\right)
\end{aligned}
$$

\section{Proposed NMCMA}

The cost function of NMCMA is the same as MCMA shown in [11] and [12]. In order to derive the NMCMA using SGD, the expectation operation is removed and the resulting cost function, $J_{N M C M A}$, is given by

$$
J_{\text {NMCMA }}(n)=J_{R}(n)+J_{I}(n)
$$

where

$$
J_{R}(n)=\frac{1}{4}\left[\left(\left|y_{R}(n)\right|^{2}-R_{2, R}\right)^{2}\right]
$$

and

$$
J_{I}(n)=\frac{1}{4}\left[\left(\left|y_{I}(n)\right|^{2}-R_{2, I}\right)^{2}\right] .
$$

Similar to the derivation in NCMA, considering $w_{p}$, we have

$$
\begin{aligned}
\frac{\partial J_{N M C M A}(n)}{\partial w_{p}} & =\left(\left|y_{R}(n)\right|^{2}-R_{2, R}\right)\left|y_{R}(n)\right| \frac{\partial y_{R}(n)}{\partial w_{p}} \\
& +\left(\left|y_{I}(n)\right|^{2}-R_{2, I}\right)\left|y_{I}(n)\right| \frac{\partial y_{I}(n)}{\partial w_{p}},
\end{aligned}
$$

Where

$$
\begin{aligned}
\frac{\partial y_{R}(n)}{\partial w_{p}} & =\frac{\partial y_{R}(n)}{\partial w_{p, R}}+j \frac{\partial y_{R}(n)}{\partial w_{p, I}} \\
& =f^{\prime}\left(n e t_{R}(n)\right) x_{R}(n-p)-j f^{\prime}\left(n e t_{R}(n)\right) x_{I}(n-p)
\end{aligned}
$$

and

$$
\begin{aligned}
\frac{\partial y_{I}(n)}{\partial w_{p}} & =\frac{\partial y_{I}(n)}{\partial w_{p, R}}+j \frac{\partial y_{I}(n)}{\partial w_{p, I}} \\
& =f^{\prime}\left(n e t_{I}(n)\right) x_{I}(n-p)-j f^{\prime}\left(\operatorname{net}_{I}(n)\right) x_{R}(n-p) .
\end{aligned}
$$

Organizing (19)-(21), and assuming $\mu$ as the learning rate, the weights update from the $n$th step to the $(n+1)$ th step can be expressed as

$$
W(n+1)=W(n)-\mu e(n) X^{*}(n),
$$

where

$$
e(n)=e_{R}(n)+j e_{I}(n),
$$

where

$$
e_{R}(n)=\left(\left|y_{R}(n)\right|^{2}-R_{2, R}\right)\left|y_{R}(n)\right| f^{\prime}\left(\operatorname{net}_{R}(n)\right)
$$

and

$$
e_{I}(n)=\left(\left|y_{I}(n)\right|^{2}-R_{2, I}\right)\left|y_{I}(n)\right| f^{\prime}\left(\text { net }_{I}(n)\right) .
$$

\section{Computer Simulations}

The proposed NCMA and NMCMA are demonstrated by using the 16-QAM symbols through a multipath channel. Their performances are compared to those of the preexisting CMA and MCMA. A typical complex-valued 10-path communication propagation channel, labeled $H(z)$ [18], is used in this simulation, which is given by

$$
\begin{aligned}
& H(z)=(0.0410+j 0.0109)+(0.0495+j 0.0123) z^{-1} \\
& +(0.0672+j 0.0170) z^{-2}+(0.0919+j 0.0235) z^{-3} \\
& +(0.7920+j 0.1281) z^{-4}+(0.3960+j 0.0871) z^{-5} \\
& +(0.2715+j 0.0498) z^{-6}+(0.2291+j 0.0414) z^{-7} \\
& +(0.1287+j 0.0154) z^{-8}+(0.1032+j 0.0119) z^{-9}
\end{aligned}
$$

We set signal-to-noise ratio (SNR) to $30 \mathrm{~dB}$ for all cases. For 16-QAM symbols, the constant modulus, $R_{2}$, is equal to 13.2. The real component, $R_{2, R}$, and the imaginary component, $R_{2, I}$, are both equal to 8.2. The length of NCMA, $M$, is set as 15 , the same as CMA. The length of NMCMA, $M$, is set as 21 , the same as MCMA. The adaptation step-size is set as 0.00001 for all. All initialized weights are set randomly with small values of approximately $10^{-5}$ except for the center weights, 
which are set as $1.0+j 0$. The $\operatorname{NTF} f(x)=x+$ $\alpha \sin (2 \pi f x)$ is used, where the nonlinear coefficient $\alpha$ is set as 0.3 and the frequency of the sine function, $f$, is chosen as $\frac{1}{4 \sqrt{R_{2}}}$ for NCMA, and $\frac{1}{4 \sqrt{R_{2, R}}}$ or $\frac{1}{4 \sqrt{R_{2, I}}}$ for NMCMA.

After 12000 training symbols, the following 2000 received symbols are tested for evaluating the equalization performance. The symbols' constellation after CMA equalizer is illustrated in Figure 3, whose estimation error is comparatively large. For comparison, the symbol's constellation after NCMA equalizer is shown in Figure 4, where the equalized symbols more concentrate on their supposed position and their bias are much smaller. To be clear, the MSEs of CMA and MCMA are plotted in Figure 5. One can see that, NCMA, with the

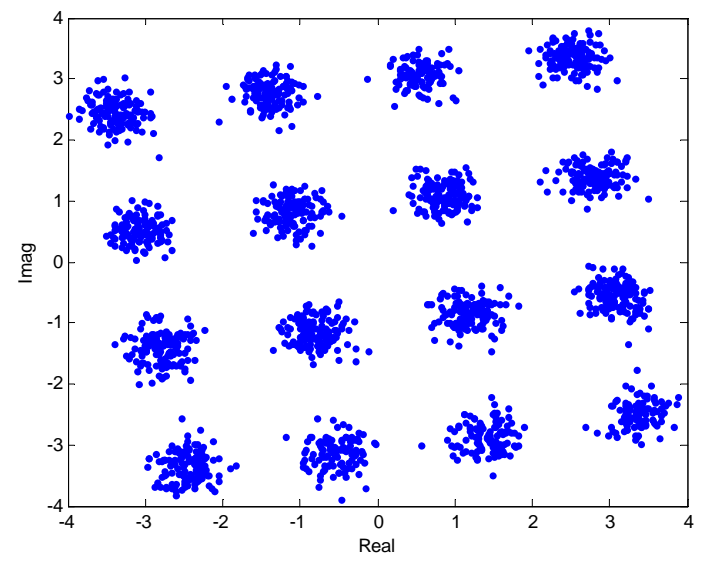

Figure 3. Constellation of 16-QAM symbols after CMA equalizer.

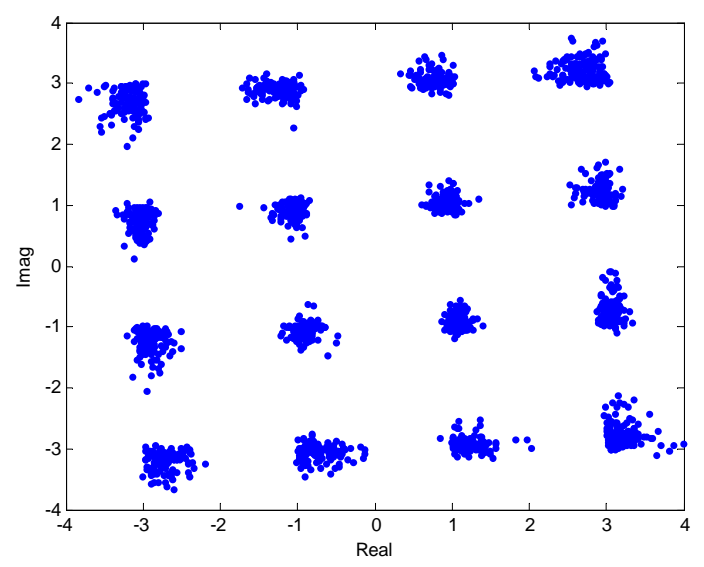

Figure 4. Constellation of 16-QAM symbols after the proposed NCMA equalizer.

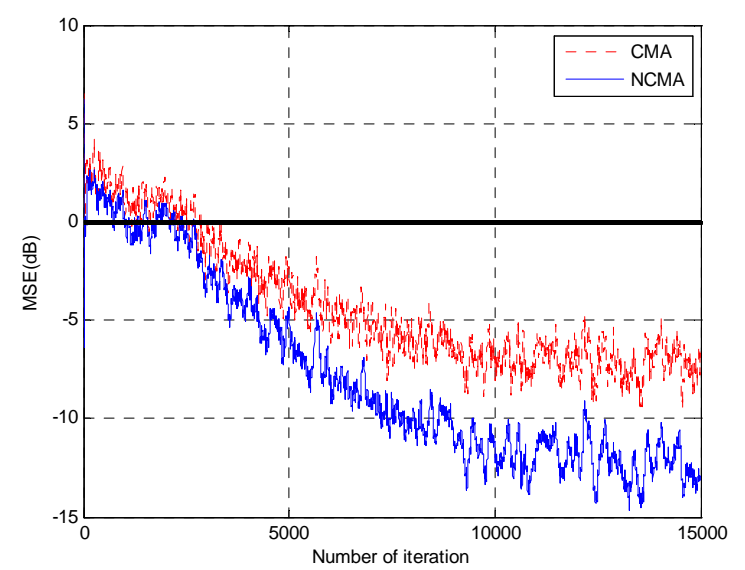

Figure 5. Performance comparison of CMA and the proposed NCMA in terms of MSE.

MSE of approximate $-13 \mathrm{~dB}$, performs better than CMA, with the MSE of approximately $-7 \mathrm{~dB}$.

Similarly, the symbols' constellation after MCMA equalizer is illustrated in Figure 6, whose estimation error is comparatively large. For comparison, the symbol's constellation after NMCMA equalizer is shown in Figure 7, where the equalized symbols much more concentrate on their supposed position and their bias are much smaller. To be clear, the MSEs of MCMA and NMCMA are plotted in Figure 8. One can see that, NMCMA, with the MSE of approximately $-30 \mathrm{~dB}$, performs better than MCMA, with the MSE of approximately $-15 \mathrm{~dB}$.

\section{Conclusions}

Two Nonlinear blind equalizers: NCMA and NMCMA, were proposed in this paper by applying the NTF into the existing CMA and MCMA, respectively. The NTF effect

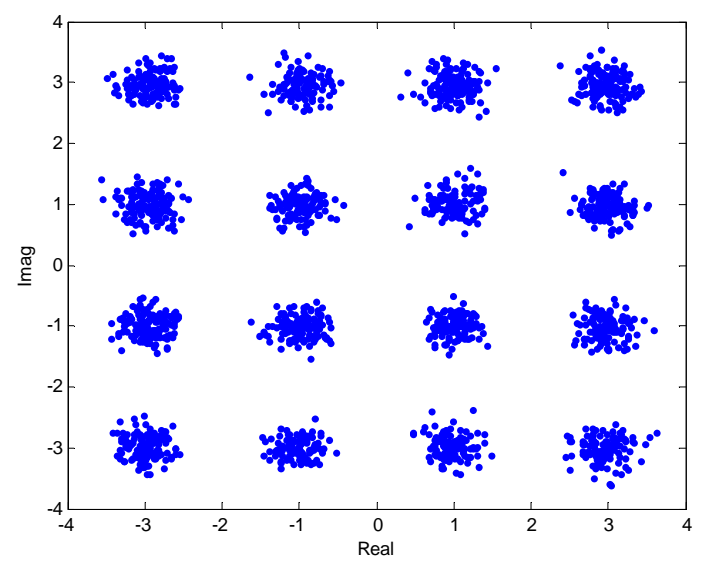

Figure 6. Constellation of 16-QAM symbols after MCMA equalizer. 


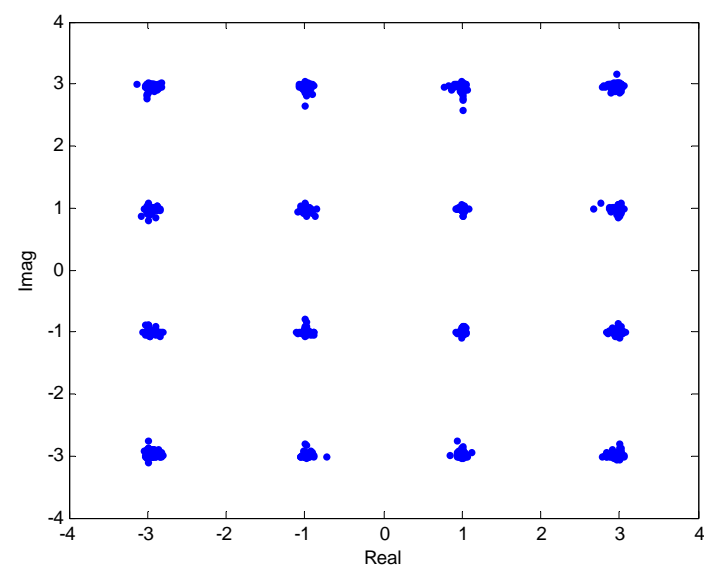

Figure 7. Constellation of 16-QAM symbols after the proposed NMCMA equalizer.

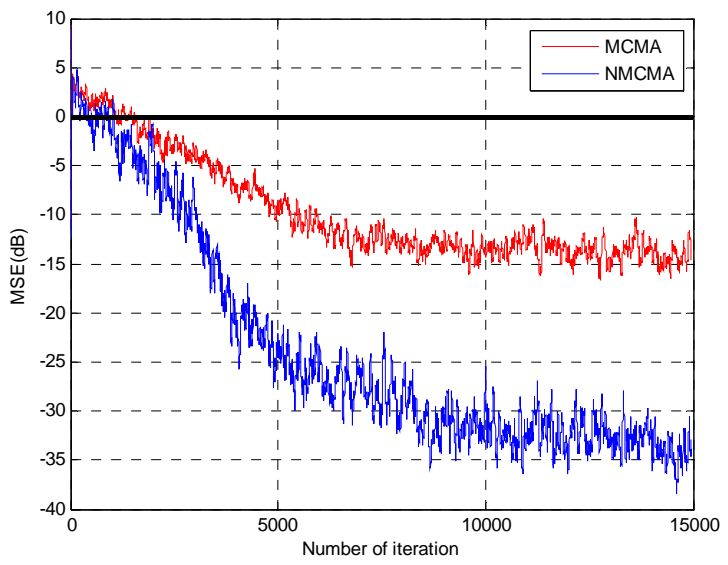

Figure 8. Performance comparison of MCMA and the proposed NMCMA in terms of MSE.

on linear blind equalizers was theoretically analyzed. It is shown that the NTF can make their decision regions sharper so that the proposed NCMA and NMCMA are more robust against the convergency error than CMA and MCMA, respectively. Computer simulations demonstrate that, for 16-QAM symbols, NCMA can reach up to approximately $-13 \mathrm{~dB}$ MSE compared with $-7 \mathrm{~dB}$ by CMA, and NMCMA can reach up to approximately -30 $\mathrm{dB}$ MSE compared with $-15 \mathrm{~dB}$ by MCMA.

\section{Acknowledgements}

The author would like to thank all the anonymous reviewers of the paper. The critical comments by all the reviewers have helped us to improve the quality of our paper.

\section{References}

[1] J. R. Treichler and B. G. Agee, "A New Approach to Multipath Correction of Constant Modulus Signals," IEEE Transactions on Acoustics, Speech, and Signal Processing, Vol. 27, No. 10, October 1979, pp. 512-530.

[2] X. H. Dai, "CMA-Based Nonlinear Blind Equalizer Modeled by a Two-Layer Feed forward Neural Network,” IEE Proceedings-Communications, Vol. 148, No. 4, April 2001, pp. 243-248.

[3] S. A. Athuraliya and L. M. Garth, "Quantized CMA Equalization for Shaped Signal Constellations,” IEEE Signal Processing Letters, Vol. 11, No. 2, February 2004, pp. 67-70.

[4] B. Baykal and A. G. Constantinides, "Matched Filtering for CMA-Based Blind Channel Estimation,” IEEE Electronics Letters, Vol. 39, No. 17, August 2003, pp. 1285-1287.

[5] D. L. Wang, H. Leung, K.-C. Kwak and H. Yoon, "Enhanced Speech Recognition with Blind Equalization for Robot 'WEVER-R2'," Proceedings of IEEE International Symposium on Robot and Human Interactive Communication, Jeju Island, August 2007, pp. 684-488.

[6] G. Gelli and F. Verde, “Two-Stage Interference-Resistant Adaptive Periodically Time-Varying CMA Blind Equalization," IEEE Transactions on Signal Processing, Vol. 50, No. 3, March 2002, pp. 662-672.

[7] K. Wesolowski, "Analysis and Properties of The Modified Constant Modulus Algorithm for Blind Equalization," European Transactions on Telecommunications, Vol. 3, No. 3, September 2008, pp. 225-230.

[8] M. Chen, Q. J. Tu and Z. Ding, "A Quadratic Programming Approach to Blind Equalization and Signal Separation," IEEE Transactions on Signal Processing, Vol. 57, No. 6, June 2009, pp. 2232-2244.

[9] Kyuho Hwang and Sooyong Choi, "Blind Equalization Method Based on Sparse Bayesian Learning," IEEE Signal Processing Letters, Vol. 16, No. 4, April 2009, pp. 315-318.

[10] I. Santamaria, C. Pantaleon, L. Vielva and J. Ibanez, "Blind Equalization of Constant Modulus Signals Using Support Vector Machines,” IEEE Transactions on Signal Processing, Vol. 52, No. 6, June 2004, pp. 1773-1782.

[11] K. Nam Oh and Y. O. Chin, "Modified Constant Modulus Algorithm: Blind Equalization and Carrier Phase Recovery Algorithm,” Proceedings of IEEE International Conference on Communications, Seattle, June 1995, pp. 498-502.

[12] M. Amin, H. Lin, C. R Reed. Jr. and R. Malkemes, “A Modified Constant Modulus Algorithm for Adaptive Channel Equalization for QAM Signals," Proceedings of IEEE Signal Processing Workshop, Cannes, August 2001, pp. 563-566.

[13] I. Chahed, J. Belzile and A. B. Kouki, "Blind Decision Feedback Equalizer Based on High Order MCMA," Proceedings of IEEE CCECE, Niagara Falls, Ontario, May 2004, pp. 2111-2114. 
[14] Z. X. Jiang, M. Zhang, Z. C. Li and S. L. Lu, "A Newly High-Speed MCMA Algorithm for QAM System," Proceedings of WiCOM, Dalian, September 2008, pp. 1-4.

[15] D. L. Wang, “A Nonlinear Modified Constant Modulus Algorithm for Blind Equalization,” Proceedings of IEEE CCECE, Calgary, May 2010, pp. 1-4.

[16] D. L. Wang and D. G. Wang, "Generalized Derivation of Neural Network Constant Modulus Algorithm for Blind Equalization," Proceedings of WiCOM, Beijing, September
2009, pp. 1-4.

[17] D. L. Wang, H. Leung, A. P. Kurian, K.-C. Kwak and H. Yoon, "A Deconvolutive Neural Network for Speech Classification with Applications to Home Service Robot," IEEE Transactions on Instrumentation and Measurement, Vol. 59, No. 9, September 2010, pp. 1297-1303.

[18] C. You and D. Hong, "Nonlinear Blind Equalization Schemes Using Complex-Valued Multilayer Feedforward Neural Networks,” IEEE Transactions on Neural Networks, Vol. 9, No. 11, November 1998, pp. 1442 -1455. 